\title{
Conceptual Exploration of Semantic Mirrors ${ }^{\star}$
}

\author{
Uta Priss, L. John Old \\ Napier University, School of Computing, \\ u.priss@napier.ac.uk, j.oldenapier.ac.uk
}

\begin{abstract}
The "Semantic Mirrors Method" (Dyvik, 1998) is a means for automatic derivation of thesaurus entries from a word-aligned parallel corpus. The method is based on the construction of lattices of linguistic features. This paper models the Semantic Mirrors Method with Formal Concept Analysis. It is argued that the method becomes simpler to understand with the help of FCA. This paper then investigates to what extent the Semantic Mirrors Method is applicable if the linguistic resource is not a high quality parallel corpus but, instead, a medium quality bilingual dictionary. This is a relevant question because medium quality bilingual dictionaries are freely available whereas high quality parallel corpora are expensive and difficult to obtain. The analysis shows that by themselves, bilingual dictionaries are not as suitable for the Semantic Mirrors Method but that this can be improved by applying conceptual exploration. The combined method of conceptual exploration and Semantic Mirrors provides a useful toolkit specifically for smaller size bilingual resources, such as ontologies and classification systems. The last section of this paper suggests that such applications are of interest in the area of ontology engineering.
\end{abstract}

\section{Introduction}

Dyvik (1998, 2003, 2004) invented the "Semantic Mirrors Method" as a means for automatic derivation of thesaurus entries from a word-aligned parallel corpus. His online interface ${ }^{1}$ uses a parallel corpus of Norwegian and English texts, from which users can interactively derive thesaurus entries in either language. A feature set is derived for each sense of each word. The senses then form a semi-lattice based on inclusion and overlap among feature sets. Priss \& Old (2004) note (without providing any details) that Dyvik's method is similar to certain concept lattices derived from monolingual lexical databases. The Semantic Mirrors Method is briefly described in section 2 of this paper. Section 3 explains how the Semantic Mirrors Method can be represented with respect to Formal Concept Analysis (FCA). We believe that the Semantic Mirrors Method is of general interest to the FCA community because there may be other similar applications in this area.

In section 4, the FCA version of the Semantic Mirrors Method from section 3 is applied to an English-German dictionary. An advantage of using bilingual dictionaries

\footnotetext{
* This is a preprint of a paper published in Ganter; Godin (eds.), Formal Concept Analysis: Third International Conference, ICFCA 2005, Springer Verlag, LNCS 3403, 2005. (c) Springer Verlag.

${ }^{1}$ http://ling.uib.no/ helge/mirrwebguide.html
} 
instead of parallel corpora is that bilingual dictionaries are freely available on the Web whereas word-aligned parallel corpora are expensive. A disadvantage of using bilingual dictionaries is that the semantic information which can be extracted from them is less complete, at least with respect to the creation of Semantic Mirrors. Therefore, in section 5 of this paper we analyse how conceptual exploration (cf. Stumme (1996)) can be used to improve the incomplete information extracted from bilingual dictionaries. Even though conceptual exploration is a semi-automated process, we believe that in combination with the Semantic Mirrors Method, this approach has potential applications with respect to ontology merging as described in section 6 .

This paper attempts to provide sufficient details of the Semantic Mirrors Method to be understandable for non-linguists, but it is assumed that readers are familiar with the basics of FCA, which can be found in Ganter \& Wille (1999).

\section{The Semantic Mirrors Method}

The Semantic Mirrors Method intends to extract semantic information from bilingual corpora, which are large collections of texts existing in two languages and which are aligned according to their translations. The assumption is that if the same sentence is expressed in two different languages, then it should be possible to align words or phrases (or "lemmata") in one language with the corresponding words or phrases in the other language. This word alignment is not trivial because languages can differ significantly with respect to grammar and syntactic ordering. Computational linguists have developed a variety of statistical algorithms for such word-alignment tasks. These algorithms perform with different degrees of accuracy. One of Dyvik's interfaces allows for users to vary the parameters used in these algorithms to explore their impact on the extracted Semantic Mirrors. For comparison, Dyvik has also experimented with manually aligned corpora ${ }^{2}$. For the purposes of this paper, only the resulting lists of aligned translations are of interest. The quality or accuracy of the word alignment algorithms are not discussed in this paper.

\subsection{Step 1}

Once a bilingual corpus is word-aligned, one can select a word in either language and list all translations of that word occurring in the corpus. These lists of words and their respective lists of translations form the basis of the Semantic Mirrors Method. Dyvik (2003) calls the set of translations of a word $a$ from language $A$ its "(first) t-image" in language $B$. One can then form the t-images (in language $A$ ) of the t-image (in language $B$ ) of word $a$ from language $A$. This set of sets is called the "inverse t-image of $a$ ". This algorithm of collecting the translations of the translations of a word has been mentioned by other authors (for example, Wunderlich (1980)) and is called the "plus operator" by Priss \& Old (2004). This algorithm presents the first step of Dyvik's Mirrors Method. In contrast to this first step which has independently been discovered by different authors, to our knowledge, the next steps of the Semantic Mirrors Method are unique to this method.

\footnotetext{
${ }^{2}$ http://ling.uib.no/ helge/mirrwebguide.html\\#bases
} 


\subsection{Step 2}

The second step is to partition the t-image of a word into distinct senses. As an example, a t-image of English "wood" in German could be \{Wald, Holz, Gehölz\}. Intuitively, these three words belong to two senses: the sense of "wood" as a collection of trees ("Wald" and "Gehölz") and the sense of "wood" as a building material ("Holz"). These senses can be derived automatically by analysing the inverse t-image, i.e., the set of sets of t-images of the initial t-image. In this example, it is assumed that the t-image of "Holz" is $\{$ timber, wood $\}$, the t-image of "Gehölz" is $\{$ grove, wood $\}$, and the t-image of "Wald" is \{grove, forest, wood $\}$. Because the t-images of "Wald" and "Gehölz" overlap in more than one word, they are considered one sense of "wood" denoted by "wood1". Because the t-image of "Holz" overlaps with the other two t-images only in the original word "wood", "Holz" is considered a second sense of "wood" denoted by "wood2".

Once each sense of a word is individuated, it can be associated with its own timage. Thus the t-image of "wood1" is $\{$ Wald, Gehölz $\}$; the t-image of "wood2" is $\{$ Holz $\}$. These two senses belong to different "semantic fields". According to Dyvik (2003): "traditionally, a semantic field is a set of senses that are directly or indirectly related to each other by a relation of semantic closeness. In our translational approach, the semantic fields are isolated on the basis of overlapping t-images: two senses belong to the same semantic field if at least one sense in the other language corresponds translationally with both of them." This means that "grove" and "forest" belong to the same semantic field as "wood1"; "timber" belongs to the same semantic field as "wood2". Of course, before assigning "grove", "forest", and "timber" to semantic fields, one would need to determine their own inverse t-images to see whether or not they have more than one sense themselves.

Dyvik (2003) explains that because the translational relation is considered symmetric, i.e. independent of the direction of the translation, one obtains corresponding semantic fields in two languages. These fields are not usually exactly structurally identical because the t-images in each language can be of different sizes and their subrelationships can be different. But each semantic field imposes a subset structure on the corresponding semantic field in the other language. Thus each semantic field is structured by its own subset relationships and receives further structures from the corresponding field in the other language. We defer a more detailed description of these relationships to the next section because they are easier to explain with the help of FCA. The Semantic Mirrors Method receives its name from the fact that the semantic structures from one language can be treated as a "Semantic Mirror" of structures in the other language.

\subsection{Step 3}

In the third step of the Semantic Mirrors Method a feature hierarchy is formed based on the set-structures. Again this is more easily explained with FCA in the next section. The idea of expressing semantic information in feature hierarchies (or lattices) is common in the field of componential semantics. But in contrast to componential semantics where features often represent abstract ontological properties (such as "material", "immaterial"), in the Semantic Mirrors Method features are automatically derived as pairs 
of senses from the two languages, such as "[wood1, Holz]". Thus there is no attempt to manually de-construct features into any form of primitives or universals.

\subsection{Step 4}

As a last step of the Semantic Mirrors Method, thesaurus entries are generated. This is achieved by operations which extract synonyms, hypernyms, hyponyms and related words for a given word. In contrast to the feature structures which are graphically displayed as lattices, the thesaurus entries are displayed in a textual format. Users of the on-line interface can vary the parameters of "SynsetLimit" and "OverlapThreshold" which influence how wide or narrow the notion of "synonymy" is cast and thus how the thesaurus entries are constructed. The FCA description in the next section does not include an analysis of Step 4 because we have not yet determined whether there is any advantage of using FCA at this stage. That will be left for future research.

\section{An FCA description of Semantic Mirrors}

Dyvik (1998) uses Venn diagrams as means of visualising and explaining the different steps of the Semantic Mirrors Method. A disadvantage of Venn diagrams is that they are difficult (or even impossible) to draw for more complex examples. This section demonstrates how concept lattices can be used to visualise the first three steps of the Mirrors Method. As Priss \& Old (2004) observe, the first step of the Semantic Mirrors Method is similar to what Priss \& Old call "neighbourhood lattices" with respect to lexical databases. By modelling the Semantic Mirrors Method with FCA, the techniques developed for neighbourhood lattices can now also be applied to the Semantic Mirrors Method and vice versa.

\subsection{Step 1: forming a neighbourhood lattice}

The first step of the Semantic Mirrors Method consists of constructing a formal context, which has a union of t-images as a set of objects and a union of corresponding inverse t-images as a set of attributes. Figure 1 shows an example for English "good", "clever", "cute" and "pretty". The data for this example comes from one of Dyvik's "toy" examples $^{3}$. The t-images (or translations) of "good", "clever", "cute" and "pretty" are the objects in figure 1. The inverse t-images (i.e., the translations of the translations) are the attributes. This kind of lattice is a "neighbourhood lattice" in the sense of Priss \& Old (2004).

Instead of t-images and inverse t-images, one can also use inverse t-images and inverse t-images of inverse t-images, and so on. In many cases the continuous search for translations may not converge until large sets of words from both languages are included. For example, Dyvik and Thunes started with the Norwegian words "god", "tak" and "selskap" . After translating back and forth between Norwegian and English four

\footnotetext{
${ }^{3}$ http://ling.uib.no/ helge/mirrwebguide.html

${ }^{4}$ http://ling.uib.no/ helge/mirrwebguide.html\\#bases
} 


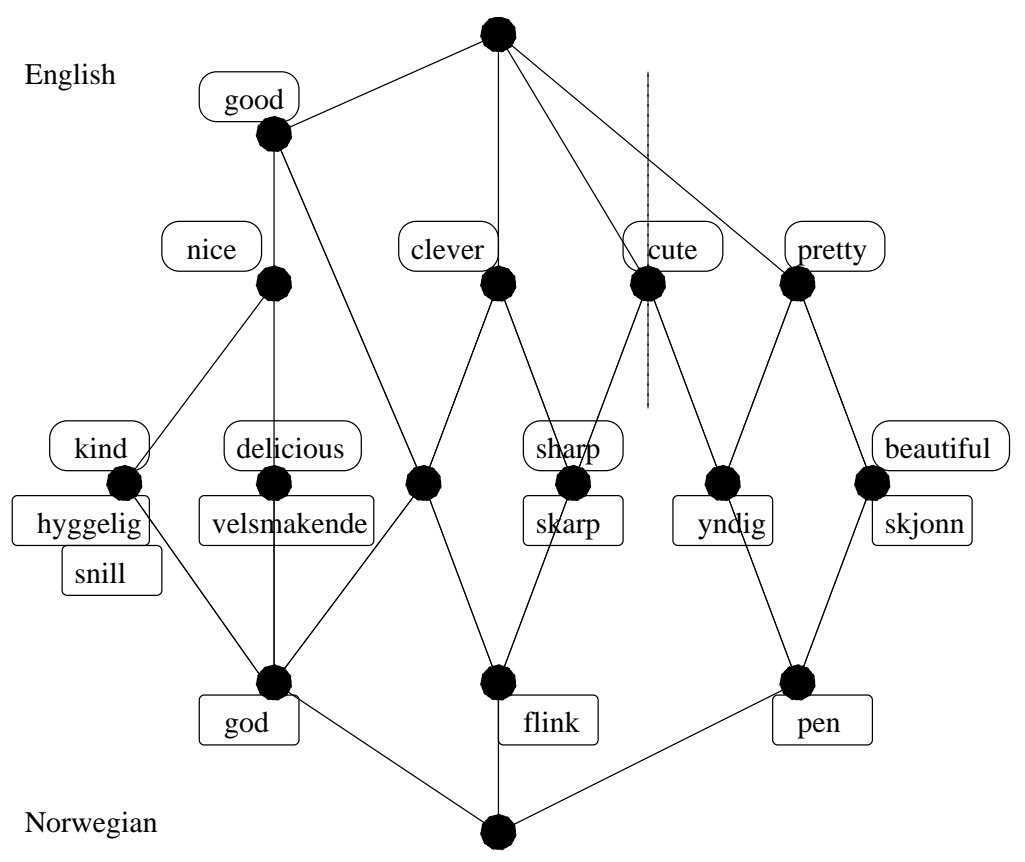

Fig. 1. A neighbourhood lattice for "good/god" (Step 1)

times, they collected a set of 2796 Norwegian words and 724 English words! Therefore, it may be sensible for some applications to terminate the search for t-images after a few iterations. The resulting neighbourhood lattice is complete for the initial set but incomplete either with respect to the translations of the objects or the attributes. If the translations are stopped after adding attributes, then some of the attributes which were added last may not have their complete set of translations among the objects. If the translations are stopped after adding objects, then some of these may be missing some of their translations.

\subsection{Step 2: the Sense Distinction Algorithm}

The second step of the Semantic Mirrors Method consists of identifying which different senses each word has. The different senses are then used to form different semantic fields. Modelled with respect to FCA, we call this algorithm the "Sense Distinction Algorithm". This algorithm can be applied to any finite formal context, but we do not know whether the algorithm produces any interesting results for other formal contexts than those describing neighbourhood lattices. It should be noted that this algorithm focuses on attributes attached to co-atoms (the lower neighbours of the top concept) and on objects attached to atoms (the upper neighbours of the bottom concept). Thus in figure 1 only the attributes "good", "clever", "cute" and "pretty" and the objects "god", "flink", and "pen" are of interest. A "contingent" of a concept is defined as the set of 
attributes and objects, which are in the extension of the concept but not in the extension of any subconcept and in the intension of the concept but not in the intension of any superconcept. Thus these attributes and objects belong directly to the concept and are not inherited from sub- or superconcepts. In line diagrams, such as figure 1, the objects and attributes attached to each node form the contingent of that concept.

The Sense Distinction Algorithm can be described as follows:

- For each co-atom $c$ which has attribute $a$ in its contingent collect the set $S$ of concepts immediately below and adjacent to $c$.

- i) If $c$ also has at least one object in its contingent, then each object in the contingent defines one sense of $a$.

- ii) If the meet of $S$ is above the bottom concept $\perp$, then $a$ has one remaining sense. Skip iii) and continue with the next co-atom.

- iii) Else, if the meet of $S$ is the bottom concept, construct a relation $R$ as follows: for $c_{1}, c_{2} \in S: c_{1} R c_{2}: \Longleftrightarrow c_{1} \wedge c_{2}>\perp$. Form the transitive closure of $R$ (which makes $R$ an equivalence relation on $S$ ). The remaining senses of $a$ now correspond to the equivalence classes of $R$ on $S$.

- Determine the senses for each atomic object in an analogous, dual manner.

Step i) of the algorithm relates to what was said above about the incompleteness of the neighbourhood lattices. If an object is attached to a co-atom then the chances are that some of its translations are missing from the formal context. This is because many words have more than one translation, which means that they are attached to the meet (or dually join) of several co-atomic (or dually atomic) concepts. Objects that are attached to a co-atom because their translational information is incomplete, would move further down in the lattice if their translations were added to the set of attributes. Therefore objects attached to co-atoms can indicate that the word which is the attribute of that co-atom has several senses. Step 1 provides information about whether the set of objects or whether the set of attributes may have incomplete translations. Therefore step i) can be rewritten to incorporate this information as "If $c$ has an object in its contingent and the translations of this object may be incomplete in the formal context, then each object attached to $c$ corresponds to one sense of $a$ ". But this rewritten version of step i) is different from Dyvik's (1998) Mirrors Method.

In figure 1, only the attribute "cute" has two senses. The dotted line in figure 1 indicates that the lattice contains two separate semantic fields: one for each sense of "cute". Figure 2 shows these two semantic fields. The algorithm which leads from figure 1 to figure 2 can be described as deleting the top and bottom concept and all atomic or co-atomic concepts which were identified as having words with more than one sense in the Sense Distinction Algorithm. The different senses are numerically labelled and move to adjacent concepts. For example in figure 2, "cute2" is now attached to the same concept as "sharp", and "cute1" is now attached to the same concept as "yndig". The left diagram in figure 2 is not a lattice anymore but it can be thought of as a lattice whose top and bottom concepts are omitted in the graphical representation.

\subsection{Step 3: creating mirror images}

Figure 1 shows that the neighbourhood lattice in this example is almost symmetric with respect to a horizontal line in the middle. This line can be thought of as the "Semantic 


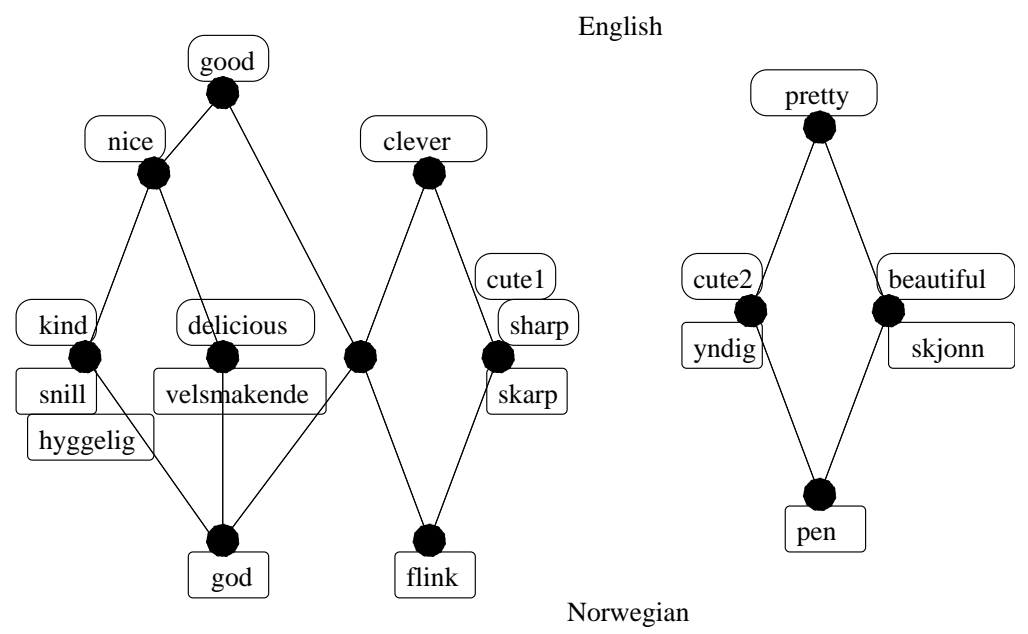

Fig. 2. Two semantic fields (Step 2)

Mirror" between the two languages. In this example, the two languages are very similar. Except for "nice", all other words have a corresponding translation in the other language. For "nice" there are two possibilities to find translations as explained further below. Figures 3 to 5 show the resulting semantic fields.

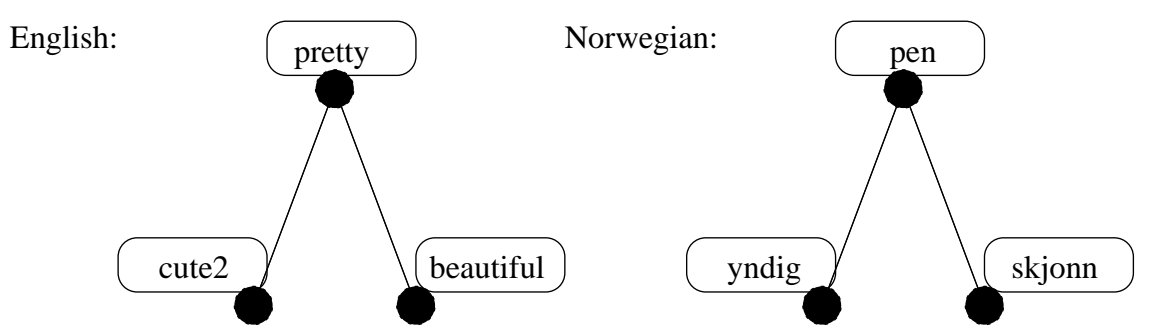

Fig. 3. The mirror images for "pretty" in English and Norwegian (Step 3)

In the example in figure 2, all attributes belong to concepts above or equal to the mirror line and all objects to concepts below or equal to the mirror line. This indicates that the lattices in this example can be cut apart along the mirror line so that each half represents the structures in one language. In general, this may not always be possible. Step 2 ensures only that co-atoms have no objects and atoms have no attributes in their contingents. The concept for "nice" could still have an object attached. It may be useful to apply the Sense Distinction Algorithm to any concept above or below the mirror line if the concept has both objects and attributes in its contingent. In any case, for each 
lattice resulting from Step 2, a "mirror" $M$ can be defined as the set of all concepts which have both objects and attributes in their contingents plus those concepts which have no objects and attributes in their contingents but which are "equi-distant" from the top and bottom concept. This notion is somewhat fuzzy because there are different possibilities for defining "equi-distance" in a lattice.

In figure 2 , the left lattice has a mirror $M$ containing 4 concepts (the anti-chain in the middle), the right lattice has a mirror $M$ containing two concepts. Each lattice is now split into two halfs as follows: a formal context $C_{1}$ is formed which has an object for each concept in the mirror $M$ and which has the original set of attributes; a second formal context $C_{2}$ is formed which also has an object for each concept in the mirror $M$ but has the original set of objects as a set of attributes. The crosses for each of these formal contexts are inserted according to the relation between the mirror elements and the objects (or attributes, respectively) in the original formal context.

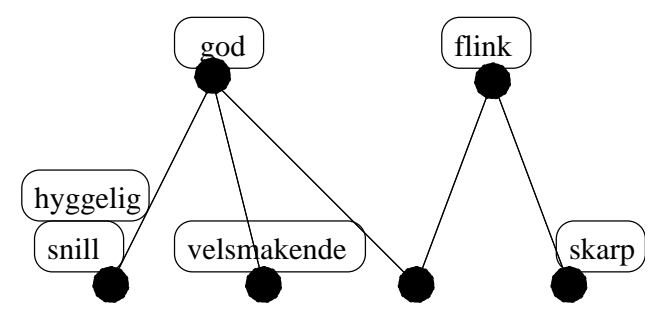

Fig. 4. The mirror image of English "good" in Norwegian (Step 3)

If $C_{1}$ and $C_{2}$ are structurally identical, then their lattices are "exact mirror images" of each other (such as in figure 3). Otherwise, Step 3 of Dyvik's method attempts to relate the concepts from $C_{1}$ and $C_{2}$ in a top-down manner, which corresponds to context isomorphisms in FCA. The resulting lattices are "distorted mirror images". If $C_{1}$ is a subrelation of $C_{2}$, then $C_{1}$ is left unchanged (such as the Norwegian "god" in figure 4). For any column in the relation of $C_{2}$ that is not contained in the relation of $C_{1}$ there are several possibilities (cf. figure 5), some of which require addition of structural attributes, which do not correspond to words in the language (such as the two missing attributes in figure 5).

\section{Semantic Mirrors in Bilingual Dictionaries}

As mentioned in the introduction, a disadvantage of the use of parallel corpora is that they are expensive to obtain or construct. On the other hand, bilingual dictionaries for many languages are available on-line for free ${ }^{5}$. These bilingual dictionaries can be of questionable quality, but methods such as the Semantic Mirrors Method should be applicable even to slightly faulty data because errors should be detectable in the end result.

\footnotetext{
${ }^{5}$ For example at www. fdicts. com
} 


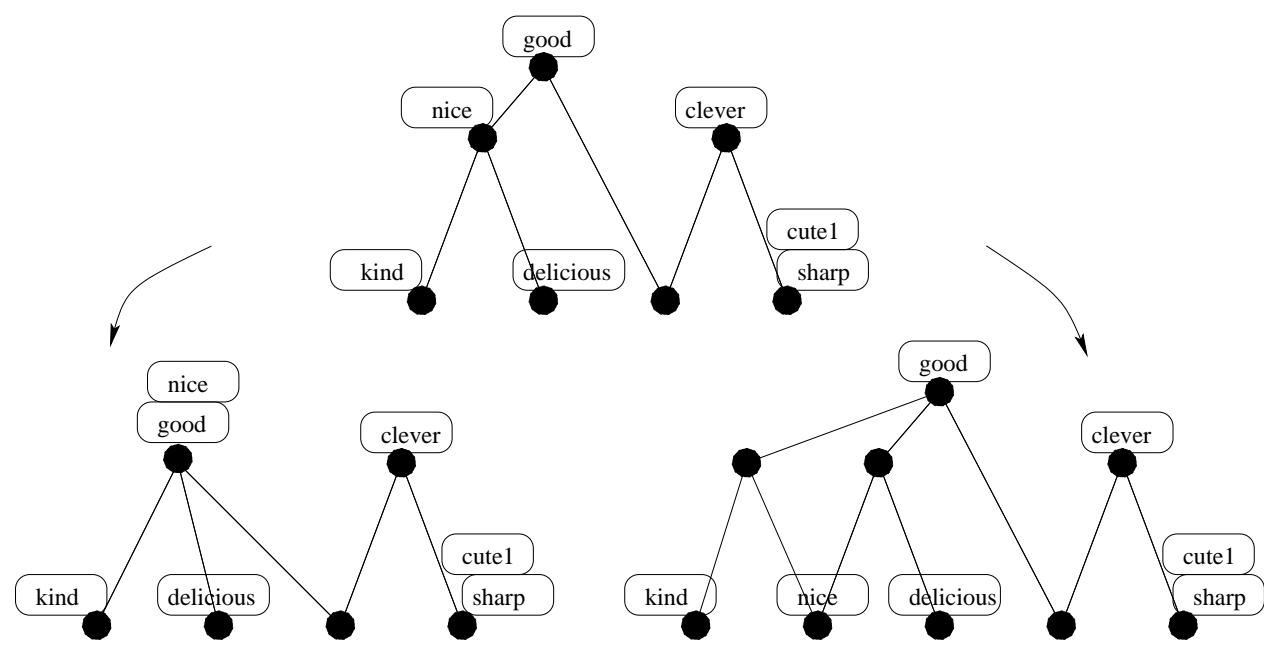

Fig. 5. The two mirror images of Norwegian "god" in English (Step 3)

The lists of possible translations which Dyvik (1998) utilises for his method are also not without errors if they are based on statistical automatic word alignment. The Semantic Mirrors Method is designed to cope with such data.

A more significant problem relating to bilingual dictionaries is not their quality but the fact that they contain fewer translations than parallel corpora because, in a corpus, words are not only translated into their direct counterparts but can also be translated into their hypernyms or hyponyms. This is because in natural language it is in general possible to use hypernyms and hyponyms for the same reference. For example, in a conversation a single person could be referred to as "the man", "that guy", "he", "Paul", and so on. Therefore in a parallel corpus of sufficient size one can expect these kinds of relationships to occur across languages. The separation into semantic fields in the Semantic Mirrors Method depends on these relationships. In a bilingual dictionary, however, it is usually attempted to translate words into exact counterparts if possible and to provide only as few translations as necessary. Therefore, one can expect that different translations of a word in a bilingual dictionary will more often refer to different senses than to synonyms within the same semantic field. For a single sense fewer translations can be expected than would be found in a parallel corpus.

The following example is constructed using a freely available German-English dictionary ${ }^{6}$. The dictionary has more than 400,000 entries and is thus of reasonable size. A manual comparison of the translations of a few words with other dictionaries shows that the dictionary is of reasonable quality. Figure 6 shows a neighbourhood lattice generated from this dictionary for the starting word "wood". This lattice is very "shallow" in that it has only two levels of concepts between the top and bottom concept. If the Sense Distinction Algorithm from the last section was applied to this lattice, every sin-

$\overline{{ }^{6} \text { http: //www }} \cdot$ dict.cc 
gle translational pair would be a separate sense. For example, "wood" would have three senses "Wald", "Gehölz", and "Holz". The resulting semantic fields would all be lattices consisting of a single concept. On the other hand, manual inspection of the lattice indicates that there are two larger fields contained among the words: one for the "set of trees"-sense of "wood" and one for the building material sense of "wood". Several words indicate other semantic fields, which are incomplete in the context, such as the "beam" sense of "timber" and the other senses of "lumber". Clearly, the Sense Distinction Algorithm is insufficient in this case because it does not result in such fields. The reason for this insufficiency, however, is not a shortcoming of the algorithm but instead the differences in the nature of the data derived from bilingual dictionaries opposed to parallel corpora.

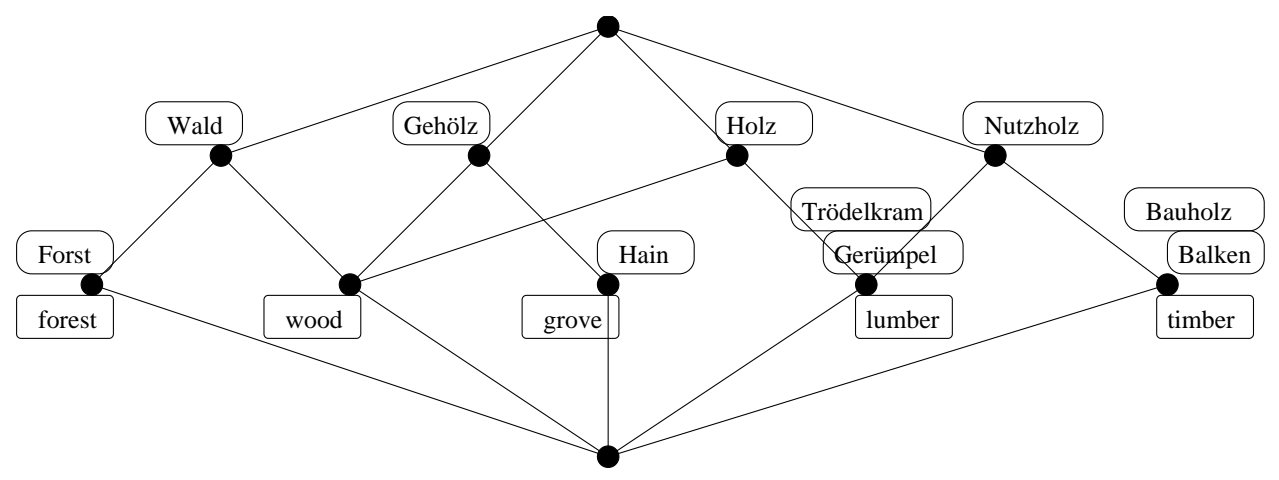

Fig. 6. A neighbourhood lattice generated from a bilingual dictionary

\section{Conceptual Exploration of Semantic Mirrors}

Since the Sense Distinction Algorithm is insufficient with respect to bilingual dictionaries, the question arises as to how it can be improved. A common FCA technique for improving incomplete data sets is "conceptual exploration". Stumme (1996) lists four cases of conceptual exploration: attribute exploration, which refers to the process of interactive addition of attributes to a formal context, object exploration, which refers to the process of interactive addition of objects to a formal context, concept exploration, which refers to the process of interactive addition of objects and attributes to a formal context, and an un-named fourth type of conceptual exploration, which refers to the process of interactive addition of crosses to a formal context. Stumme notes that so far there exists no exploration software for this last type of exploration.

The main shortcoming of the lattice in figure 6 is the fact that it is too shallow. From a linguistic view this means that hypernyms are missing. For example, "wood" in its first sense could be considered a hypernym of "grove" and "forest", but that is not depicted in the lattice. From an FCA view, for a word to be a hypernym of another word, there 
must be a subset-superset relation between the intensions or extensions. If "wood" is to become a hypernym of "grove" it must also be a translation of "Hain" and a cross for "Hain/wood" must be added in the formal context. Thus hypernyms can be established by adding certain crosses to the relation of a formal context. But these crosses cannot be randomly chosen because they must result in subset-superset relations.

Stumme's fourth type of conceptual exploration is relevant for this situation. The other types of conceptual exploration can also be relevant, because in some cases a hypernym may exist in a language but may not yet be included among the objects or attributes of the formal context in question. The conceptual exploration algorithm can work as follows: the inverse t-image is formed for each co-atomic object. In each case, a user is asked whether a hypernym in the other language can be found for the set. Since there are four co-atoms in figure 6, a user will be asked four questions. The inverse t-image of "Wald" is "Wald, Gehölz, Forst, Holz". A user might decide that "wood" is in fact a hypernym in English for this set. The inverse t-image for "Gehölz" is the set "Wald, Gehölz, Hain, Holz" and also has the English hypernym "wood". For the inverse t-images of "Holz" and "Nutzholz" no English hypernyms can be found. The first two questions result in adding "Forst/wood" and "Hain/wood" to the relation. Next, any hypernym that was found is tested against any words of the other language that have not been considered as translations. Therefore a user is asked whether "wood" can also be a translation of any of the words "Trödelkram, Gerümpel, Nutzholz, Bauholz, Balken". A user might decide that "Nutzholz/wood" and "Bauholz/wood" also need to be added.

After finishing with the objects, the lattice is recalculated. It now has only three atoms: "wood", "timber" and "lumber". Each of the three inverse t-images is checked: a German hypernym of "wood, grove, forest" is identified as "Wald"; "wood, grove, lumber" has no hypernym; a German hypernym of "wood, lumber, timber" is determined to be "Holz". The crosses "Wald/grove" and "Holz/timber" are added. As a last step, the newly found hypernyms "Holz" and "Wald" are checked against the remaining English words, which does not lead to any further added crosses. The recalculated lattice is depicted in figure 7. The Sense Distinction Algorithm applied to the lattice in figure 7 identifies two senses of "wood", two senses of "Holz", two senses of "lumber" and two senses of "timber". All other words have only a single sense. This division of senses and semantic fields is much more compatible with an intuitive notion than the initial results obtained from figure 6 .

This algorithm does not identify every possibly missing cross in the original context. It only checks for hypernyms because these are essential for the division into semantic fields. We have tested the algorithm with a few other examples. It works better in areas where hypernyms are easily identifiable, such as for concrete nouns. With respect to other types of words, more research is needed. It is hoped that a heuristic rule set can be developed that adjusts the conceptual exploration to the specific requirements of the types of semantic fields that are involved. 


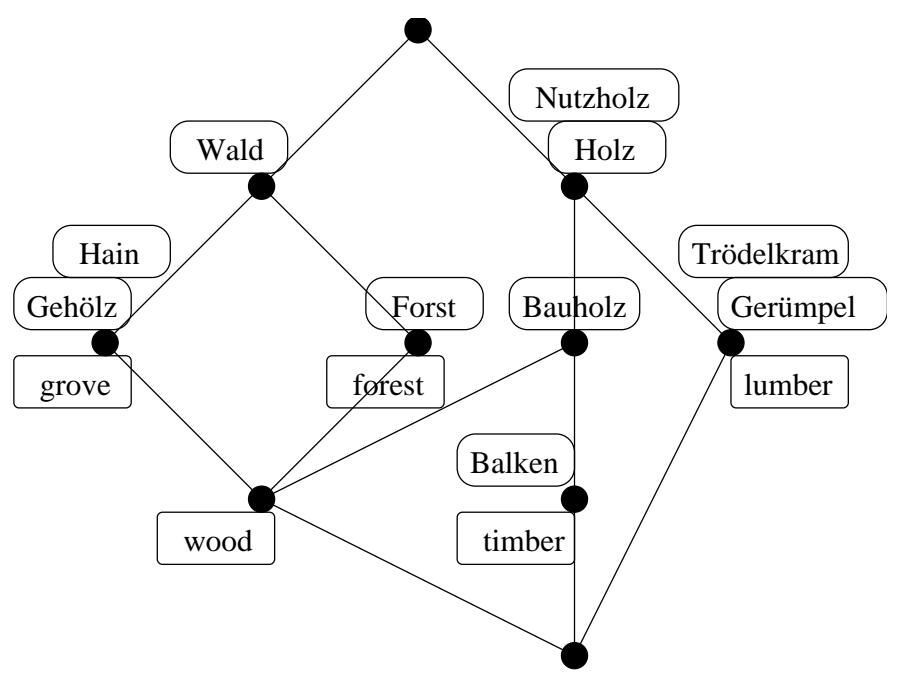

Fig. 7. The lattice after conceptual exploration

\section{Conclusion}

This paper argues that a combination of the Semantic Mirrors Method with conceptual exploration may yield promising results in certain areas. Because conceptual exploration is an interactive method, it can be labour-intensive. In the example in the last section, the relation contained 30 possibilities for added crosses, but a user was asked only 10 questions. Thus the conceptual exploration was more efficient than asking a user to manually complete a given formal context without any further tools.

We believe that these kinds of methods can be suitably applied in the area of ontology engineering and ontology merging. Ontologies are much smaller than bilingual dictionaries. While bilingual dictionaries of natural languages are too large to be processed with semi-automated methods, ontologies might still be in a range where semiautomated methods are feasible. In fact, many current methods of ontology merging in $\mathrm{AI}$ are semi-automated.

\section{Acknowledgements}

We wish to thank Helge Dyvik and three anonymous reviewers for helpful comments and suggestions.

\section{References}

1. Dyvik, Helge (1998). A Translational Basis for Semantics. In: Johansson and Oksefjell (eds.): Corpora and Crosslinguistic Research: Theory, Method and Case Studies, Rodopi, p. 51-86. 
2. Dyvik, Helge (2003). Translations as a Semantic Knowledge Source. Available on-line at www.hf.uib.no/i/LiLi/SLF/ans/Dyvik/transknow.pdf

3. Dyvik, Helge (2004). Translations as semantic mirrors: from parallel corpus to wordnet. Language and Computers, Vol. 49, iss. 1, Rodopi, p. 311-326.

4. Ganter, B.; Wille, R. (1999). Formal Concept Analysis. Mathematical Foundations. BerlinHeidelberg-New York: Springer, Berlin-Heidelberg.

5. Priss, U.; Old, L. J. (2004). Modelling Lexical Databases with Formal Concept Analysis. Journal of Universal Computer Science, Vol 10, 8, p. 967-984.

6. Stumme, Gerd (1996). Exploration Tools in Formal Concept Analysis. In: E. Diday, Y. Lechevallier, O. Opitz (Eds.): Ordinal and Symbolic Data Analysis. Proc. OSDA'95. Studies in Classification, Data Analysis, and Knowledge Organization 8, Springer, p. 31-44.

7. Wunderlich, Dieter (1980). Arbeitsbuch Semantik. Athenaeum, Königstein im Taunus. 